\title{
The application of holographic image reconstruction in the examination of liquids
}

\author{
Z. Garaguly*‡, M. Kozlovszky ${ }^{\dagger \ddagger}$, and L. Kovács ${ }^{\dagger \S}$ \\ *Doctoral School of Applied Informatics and Applied Mathematics, Obuda University, Budapest, Hungary \\ $\dagger$ John von Neumann Faculty of Informatics, Obuda University, Budapest, Hungary \\ ${ }_{\ddagger}^{\ddagger}$ BioTech Research Center, University Innovation and Research Center, Obuda University, Hungary \\ §Physiological Research Center, University Innovation and Research Center, Obuda University, Hungary
}

\begin{abstract}
With holographic microscopy, high-resolution digital images can be taken of smaller objects. The technology exploits the wave feature of light in a way that even a threedimension image can be taken, therefore, the examined objects spatial structure can also be defined. The holographic application can eliminate one of the biggest deficiency of microscopes, the depth of focus [1]. Therefore, it can be applied at processes, in which the examined objects shape, extension may carry additional information. Such processes can be, for instance, the examination of liquids, or blood. Through the process of liquid-examination, the shaped elements spatial extension can be allocated as well.
\end{abstract}

\section{INTRODUCTION AND OBJECTIVES}

The digital images of objects and shaped elements are taken by a self-made digital holographic microscope. These holographic images are processed by an also self-developed software. Through the process, the examined objects different parameters can be defined, whereas at the end of the process a more illustrative three-dimension image can be generated, in which the objects spatial extension can be observed well. The different parameters of the objects can be defined more clearly with the gained additional information, hence, in case of a blood test, for example, the diagnosis can be set more precisely. The additional information is crucial in carrying out a diagnosis.

The main objective was to develop an imaging or image reconstruction method, by which the holographic images of liquids could be processed. Processing means that the shaped elements in the liquid should be transformed into countable, recognizable and visual form. It consists of several parts. At first, it should be determined how big the seen item is, then its shape and spatial extension should be defined. The spatial extension can be easily visualised. A three-dimension model should be created of the shaped element of the holographic image and then this should be depicted. Knowing the spatial structure can mean additional information in some cases, in opposition to two-dimension data.

The image reconstruction methods necessary for liquid examination have been introduced in previous publications [2], [3], although their brief summary can be found in the following chapters.

Numerous international publications give overall review of holographic image reconstruction processes [4], [5], [6], [7], [8]. In the following the principle of hologram creation and numeric reconstruction as well as the Fresnel approximation is presented.

\section{Digital hologram PREPARATION AND THE PRINCIPLE OF NUMERIC RECONSTRUCTION}

The principle of the digital holograms preparation is that an interference is created on the image sensors surface of CCD or CMOS, which evolves in between the reference light wave and the one reflected by the object. Therefore, the interference image- the hologram- is recorded by the sensor electronically. The examined object can be a three-dimension body, or liquid which reflects, hence its examined surface is situated distance $d$ from the sensor. At the reconstruction, the real and virtual image are also placed distance $d$ from the sensor but the real image is presented in reversed position, shown in the Fig. 1. [9], [10].

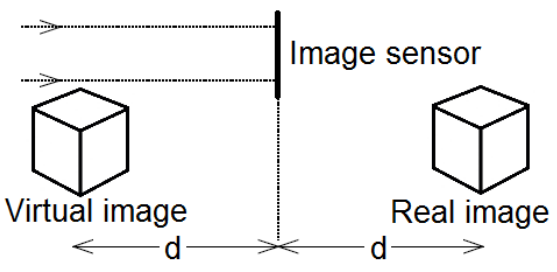

Fig. 1. Sensor, real and virtual image location.

The light waves deviation/diffraction is taken place through an aperture system of slot, which is placed perpendicularly in relation to the incoming light. This correlation is described by the Fresnel-Kirchhoff diffraction integral (1) [5], [6].

$$
\begin{gathered}
\Gamma\left(\xi^{\prime}, \eta^{\prime}\right)=\frac{i}{\lambda} \int_{-\infty}^{\infty} \int_{-\infty}^{\infty} h(x, y) E_{R}(x, y) \frac{\exp \left(-i \frac{2 \pi}{\lambda} \rho^{\prime}\right)}{\rho^{\prime}} d x d y \\
\rho^{\prime}=\sqrt{\left(x-\xi^{\prime}\right)^{2}+\left(y-\eta^{\prime}\right)^{2}+d^{2}}
\end{gathered}
$$

$H(x, y)$ is the holograms function and, $\rho^{\prime}$ is the distance between the a point of the holograms plane and a point of the reconstructions plane. However, this integral is difficult to evaluate, even with numerical and analytical methods. Approximations should be introduced in order to simplify the method [11]. 


\section{NUMERICAL RECONSTRUCTION (FRESNEL APPROXIMATION)}

The Fresnel-Kirchhoff diffraction integral can be expanded to a Taylor series if the distance between $(x, y)$ and $(\xi, \eta)$ is smaller than the distance between the reconstruction plane and the camera, the $d$ distance. After the organisation of values, it can be seen that $\rho$ distance will consist of square and linear members (3) [7].

$$
\rho=d+\frac{(\xi-x)^{2}}{2 d}+\frac{(\eta-x)^{2}}{2 d}
$$

Applying and simplifying the approximation, we get the equation of the Fresnel approximation, also known as Fresnel transformation (4) [12].

$$
\begin{array}{r}
\Gamma(\xi, \eta)=\frac{i}{\lambda d} \exp \left(-i \frac{2 \pi}{\lambda} d\right) \exp \left[-i \frac{\pi}{\lambda d}\left(\xi^{2}+\eta^{2}\right)\right] \times \\
\times \int_{-\infty}^{\infty} \int_{-\infty}^{\infty} E_{R}^{*}(x, y) h(x, y) \exp \left[-i \frac{\pi}{\lambda d}\left(x^{2}+y^{2}\right)\right] \\
\exp \left[i \frac{2 \pi}{\lambda d}(x \xi+y \eta)\right] d x d y
\end{array}
$$

Since this equation is mathematically similar to the Fourier transformation, the reconstruction of the wave field in one plane is possible. This plane will be the real image plane. On this basis, the intensity and phase can also be defined. The formula, used for producing the real image, will be the following after the simplification [11]:

$$
\begin{array}{r}
\Gamma(\nu, \mu)=\frac{i}{\lambda d} \exp \left(-i \frac{2 \pi}{\lambda} d\right) \exp \left[-i \pi \lambda d\left(\nu^{2}+\mu^{2}\right)\right] \times \\
\times \int_{-\infty}^{\infty} \int_{-\infty}^{\infty} E_{R}^{*}(x, y) h(x, y) \exp \left[-i \frac{\pi}{\lambda d}\left(x^{2}+y^{2}\right)\right] \\
\exp [i 2 \pi(x \nu+y \mu)] d x d y
\end{array}
$$

The resulting correlation and the application of the inverse Fourier transformation further simplifies the current equation. It should be interpreted as a further condition that the hologram functions $h(x, y)$ sampling derives from an $N x N$ point net, along with the coordinates of step $\Delta x$ and $\Delta y$. Values $\Delta x$ and $\Delta y$ are the image sensors pixel distance towards $x$ and $y$ direction. The previously determined integral can be transformed into finite amounts if the previous condition is applied. These finite amounts can be further simplified in accordance with the theory of Fourier transformation which is the following [13]:

$$
\Delta \nu=\frac{1}{N \Delta x} ; \quad \Delta \mu=\frac{1}{N \Delta y} ;
$$

Matrix $\Gamma$ can be calculated with the inverse discrete transform of the product of $E_{R}^{*}(k, l), h(k, l)$ and $\exp \left[-i \pi /(\lambda d)\left(k^{2} \Delta x^{2}+l^{2} \Delta y^{2}\right)\right]$. The calculation can be accelerated by the Fast Fourier Transformation. Having done this, we get the equation of the Fresnel approximation, which can be used for the reconstruction (7) [13]:

$$
\begin{array}{r}
\Gamma(m, n)= \\
=\frac{i}{\lambda d} \exp \left(-i \frac{2 \pi}{\lambda} d\right) \exp \left[+i \pi \lambda d\left(\frac{m^{2}}{N^{2} \Delta x^{2}}+\frac{n^{2}}{N^{2} \Delta y^{2}}\right)\right] \times \\
\times \sum_{k=0}^{N-1} \sum_{l=0}^{N-1} E_{R}(k, l) h(k, l) \exp \left[+i \frac{\pi}{\lambda d}\left(k^{2} \Delta x^{2}+l^{2} \Delta y^{2}\right)\right] \\
\exp \left[i 2 \pi\left(\frac{k m}{N}+\frac{l n}{N}\right)\right]
\end{array}
$$

\section{INTRODUCTION OF THE TESTING ENVIRONMENT}

Data of testing environment:

- Camera - Alpha DCM510 microscope camera

- Pinhole - $10 \mu m$ hole

- Source of light - Blue LED light (470nm)

The main part of testing environment is the self-developed inline holographic microscope, which structure is shown on Fig. 2 [14].

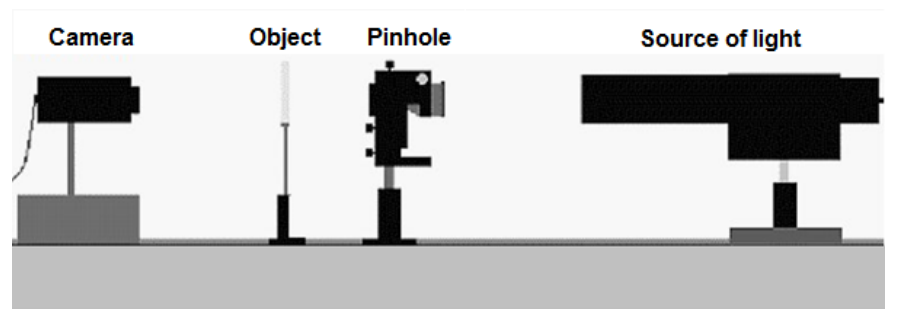

Fig. 2. Inline holographic microscope

A great advantage of inline construction is that the parts which participate in the image creation are completely situated in one line. Consequently, the source of light functions in the role of the object and the reference bar at once. It is also important to mention that the installation is a system without lens. The source of light at the tests were an RGB led, and its $470 \mathrm{~nm}$ blue light supposed to be the most ideal for the records. These light bars reach the object through a pinhole, then these reach the camera. The applied pinhole was a $10 \mu m$ one. In the case, the pinhole is a small metal disk, with a $10 \mu \mathrm{m}$ hole in its middle. The light bars can go through only this little hole. At the recording, the light, coming from the object is recorded. The recording takes place with CCD or CMOS image sensor. The product of the process is the holographic image of the object, also called hologram. At testing, the recordings were made by an Alpha DCM510 microscope camera, without any lens. The testing environment was prepared for liquid examination, but, previously, calibration and measurement had been necessary. At this calibration process images were taken from USAF 1951 resolution testing plate (Fig. 3). The device can be calibrated precisely on the basis of these records, as well as the resolution and sizing can be also determined, since the size of the objects which can be seen on the resolution testing plate are known [14]. 


\section{2 三 III $\stackrel{1}{1 \text { IIIIE } 1}$ \\ 3 三III ${ }^{2}{ }^{3}$ III 3 \\ 3 三 \\ 4 三 III 就为 \\ 5 三III \\ $6 \equiv$ III}

Fig. 3. USAF 1951 resolution test plate

\section{MEASUREMENT DATA OF USAF 1951 RESOLUTION TESTING PLATE}

The following measurements has been made with the previously introduced processes and in the introduced testing environment.

\section{A. Camera - object distance: $3.5 \mathrm{~mm}$}

In the first setup, the distance of the camera and the object (USAF 1951 resolution testing plate) was $3.5 \mathrm{~mm}$. On the following pictures, the measurements with these settings can be seen Fig. 4 and Fig. 5. The Fresnel-method was used at the process of reconstruction.

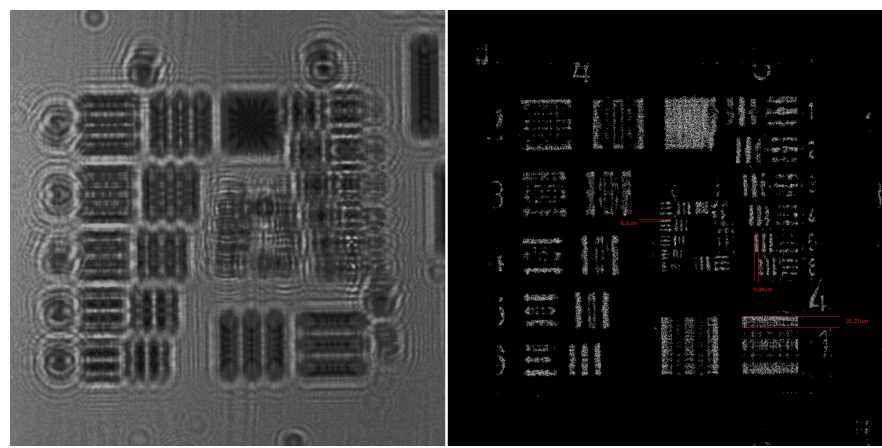

Fig. 4. Holographic and reconstructed image(USAF 1951 class: 4, 5, distance: $3.5 \mathrm{~mm})$

\section{B. Camera - object distance: $4.5 \mathrm{~mm}$}

In the second setup, the distance between the camera and the object was $4.5 \mathrm{~mm}$. The Fresnel method was applied here as well, at the process of reconstruction. The distance value at this measurement was formed which is based on previous experiences. Besides this setting, the resulting values can be seen on Fig. 6 and Fig. 7.

Regarding the testing/calibration results, the second setup was more successful as a better resolution can be achieved, hence the smaller objects are perceivable.

\section{SHOOTING OF LIQUID (CAPILLARY TUBE)}

At this method of measurement, the modification of hardware was also necessary, since the examined liquid should

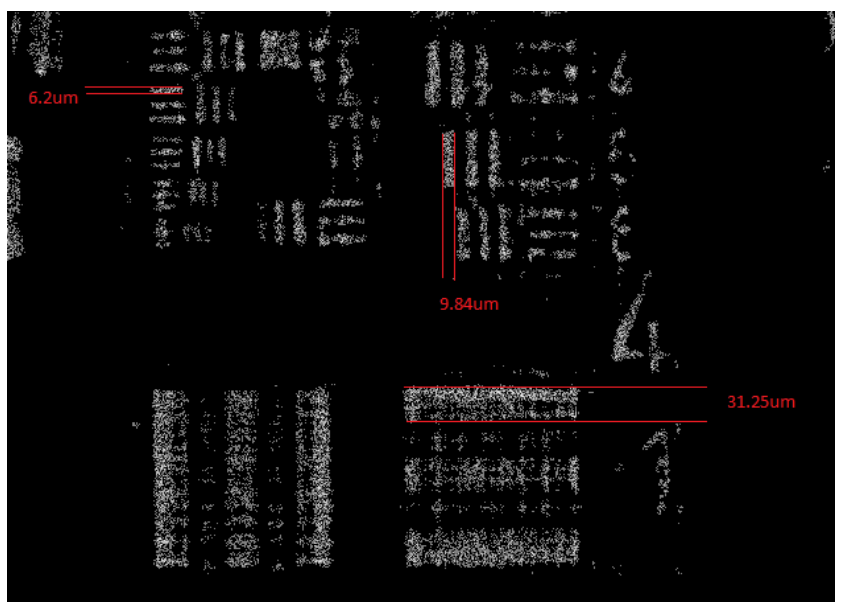

Fig. 5. Reconstructed image (USAF 1951, class: 4, 5, distance: 3.5mm) with sizing, enlarged

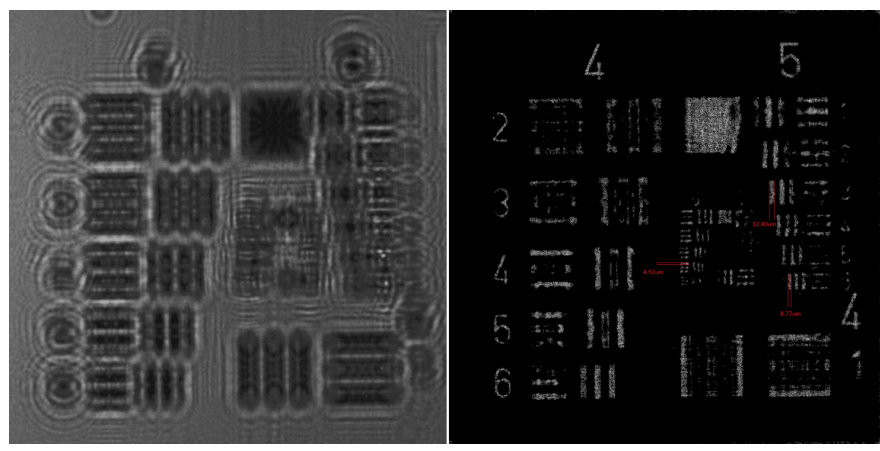

Fig. 6. Holographic and reconstructed image(USAF 1951 class: 4, 5, distance: $4.5 \mathrm{~mm})$

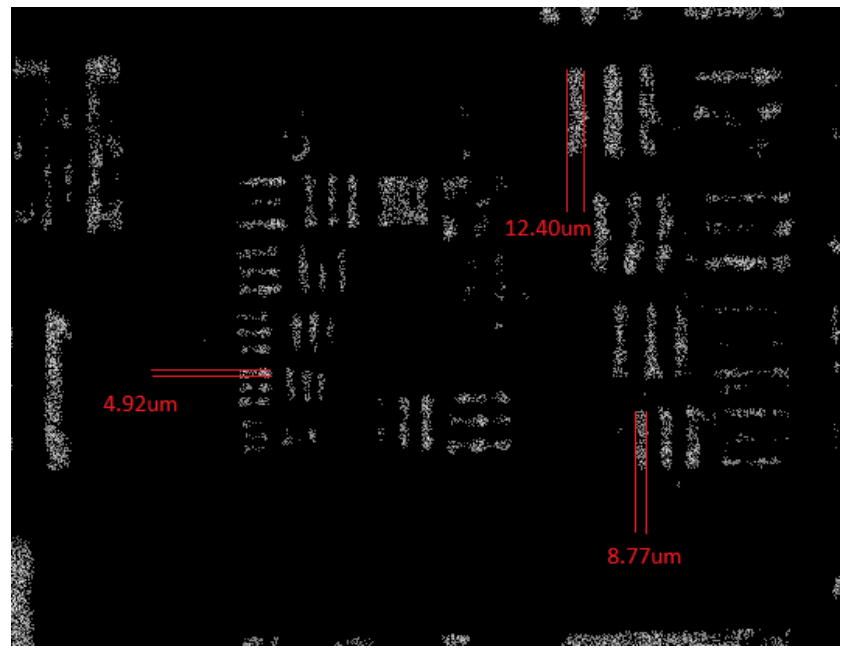

Fig. 7. Reconstructed image (USAF 1951, class: 4, 5, distance: $4.5 \mathrm{~mm}$ ) with sizing, enlarged

have been led into a device which has the function of carrying liquids. Then, it was needed to be recorded and processed. Therefore, a capillary tube was built in, replacing the slide case in the self-developed holographic microscope, afterwards 
the chance was given to examine the liquid. The inner diameter of the capillary tube is $1 \mathrm{~mm}$, its wall thickness is $0.2 \mathrm{~mm}$. The shooting was made from a solution that contained polystyrene particles diluted with distilled water, in which $4-5 \mu \mathrm{m}$ diameter polystyrene particles were placed. Due to the size of the particles and the inner diameter of the capillary tube, it caused further difficulties that several particles covered each other. This problem could be corrected for some extent, by diluting the solution. Furthermore, these polystyrene particles tend to stick together, which could be seen on the recording. As the images were taken of particles which were smaller or were at the lower bound of the hardware and the softwares range resolution, they are restricted to use for further adaptation, measure.

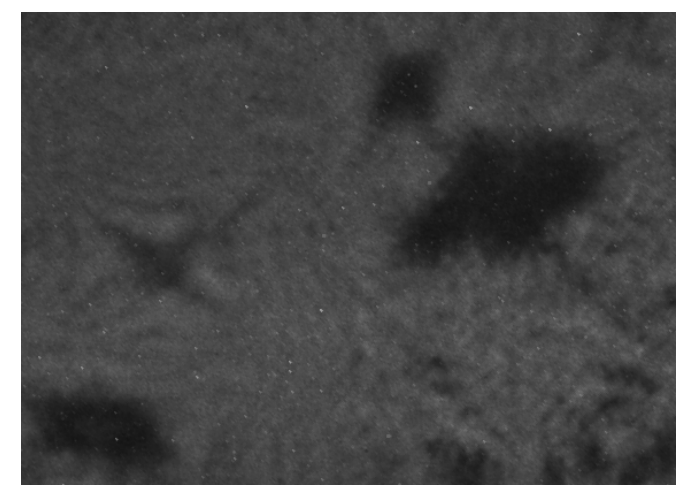

Fig. 8. Holographic image (liquid, capillary tube)

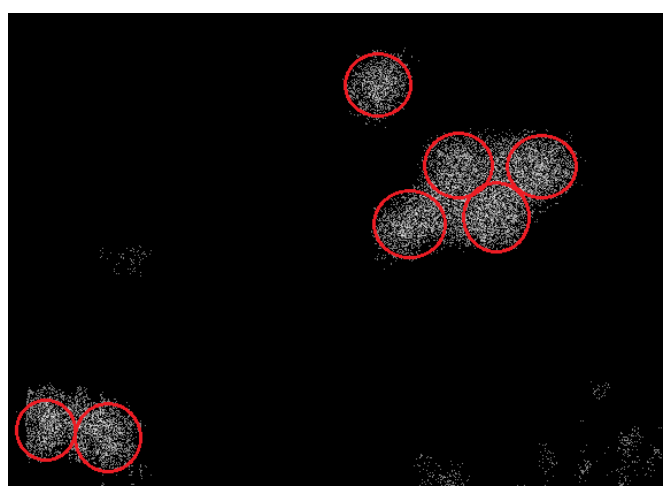

Fig. 9. Reconstructed image (liquid, capillary tube) indicated

In the previous graphs, on the reconstructed images, the marked, clumped particles can be seen. These are heavily clumped particles which make further adaptation, measures difficult as it is not easy to define that where can the border of each particle be found. Consequently, on its 3D delineation the isolation of particles cannot be seen in some cases either.

The clumping particles, which is a unique feature of this material should be taken into consideration, as in a liquid which later will be applied blood shaped elements can be found. These also tend to stick together. In order to eliminate this problem another type of examination method is described in the next chapter.

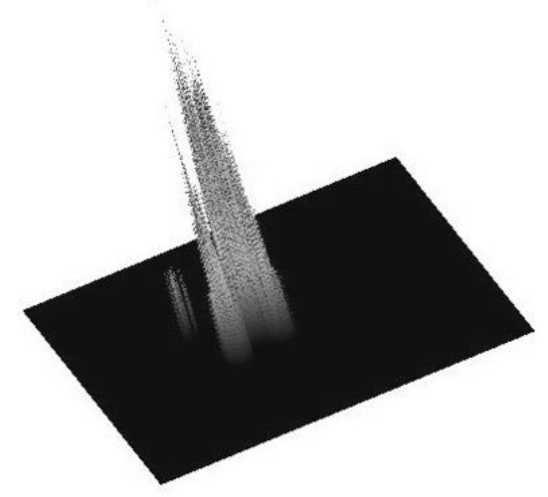

Fig. 10. Spatial structure

\section{ShOOTING OF LIQUID (SLIDE)}

At this method, images were also taken of the liquid applied at the previous examination process, but here, the images were not taken through the capillary tube. At this process, the liquid was placed onto a slide, and holographic images were taken of it. Therefore, the overlapping of particles can be fully eliminated, but it is still apparent apart from that they are tended to stick together, however, it does not influence the quality of the reconstruction. In the reconstructed image, the particles are situated well-isolated, its shape is easily recognizable. It is because the wall of the capillary tube if minimally- though it is very thin $(0.2 \mathrm{~mm})$ works as a lens, hence it distorts the image. But since the light waves coming from illumination go through the tubes completely symmetric walls, it does not distort the measurement results, however, it worsens the image quality. The image quality is worsened because the light waves of the illumination permeates the capillary tube, even the liquid itself, by weakening it further. In opposition, the surface of the slide is completely plain, hence the refraction and the weakening are minimal. Therefore, it does not affect image quality in such great extent, so a more contrasting, sharper holographic image can be taken. In the following, a holographic image can be seen, which was made by this process, and its reconstructed version (Fig. 11).

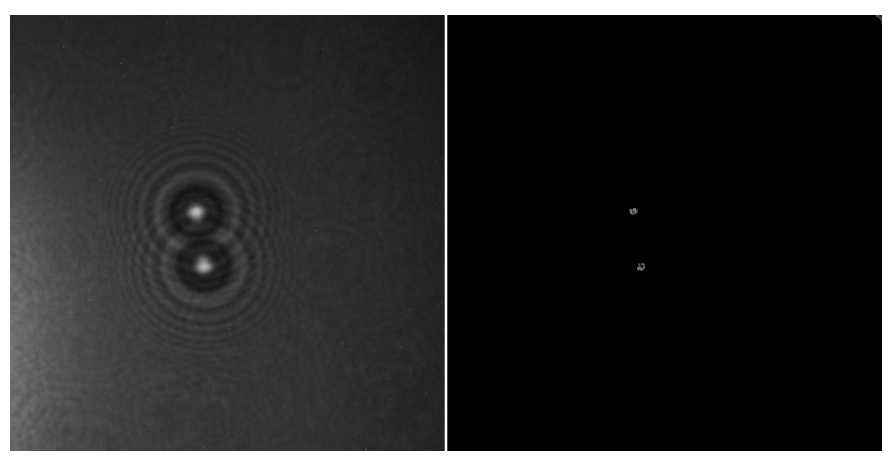

Fig. 11. Holographic and reconstructed image (liquid, slide) 


\section{CONCLUSION}

After test measurements, it was seen that the combined resolution of the hardware and software has reached that level, at which visible image can be created of $5 \mu \mathrm{m}$ objects. After these results, liquid examination could be the next step. The system was applied in two types of measurement environment, so, there were holographic images taken, and their reconstructed versions. Images were taken of liquids, which contained $4-5 \mu \mathrm{m}$ of particles. The results of the two measure methods differ highly. In the images taken of liquids in the capillary tube, it is seen that these are without contrast, and less detailed. In the other images, taken of liquid on the other slide, the same particles have appeared in a more isolated, sharper way. These measurements can be concluded in that image quality is heavily influenced by the kind of device which carries the liquid and the amount of liquid to be permeated by the light bars. The following steps will focus on that the examined medium should be formed in a way that it would be the most optimal.

\section{ACKNOWLEDGEMENTS}

The authors would like to thank University Innovation and Research Center, Obuda University, Hungary (EKIK) and the Doctoral School of Applied Informatics and Applied Mathematics, Obuda University, Hungary.

\section{REFERENCES}

[1] Th. Kreis. Holographic Interfevometry: Principles and Methods Akademie-Verlag, Berlin, and VCH Publishers, Inc., New York, 1996.

[2] Z. Garaguly, M. Kozlovszky, L. Kovács. High resolution digital holographic microscope and image reconstruction. INES 2015, 179-184, 2015

[3] Z. Garaguly, M. Kozlovszky, L. Kovács. Comparison of numerical image reconstruction methods in holography. Neumann Colloquium 2017, 6570, 2017.

[4] T. Shimobaba, N. Masuda, Y. Ichihashi and T. Ito, Real-time digital holographic microscopy observable in multi-view and multi-resolution. J. Opt. 12, 065402 (4pp) 2010.

[5] J. W. Goodman. Introduction to Fourier Optics, McGraw-Hill Companies, Inc., New York, Second Edition 1996.

[6] C. Mann, L. Yu, C.-M. Lo, and M. Kim, High-resolution quantitative phase-contrast microscopy by digital holography. Opt. Express 13, 86938698, 2005.

[7] G. Pedrini, Y. L. Zou, and H. J. Tiziani. Digital double-pulsed holographic interferometry for vibration analysis. J. Mod. Opt., 42(2), 367-374, 1995.

[8] U. Schnars, Th. M. Kreis, and W. P. 0. Jptner. Digital recording and numerical reconstruction of holograms: reduction of the spatial frequency spectrum. Opt. Eng., 35(4), 977-982, 1996.

[9] X. Yu, J. Hong, C. Liu, and M. K. Kim, Review of digital holo-graphic microscopy for three-dimensional profiling andtracking. Opt. Eng. 53, 112306, 2014.

[10] S. Teeranutranont and K. Yoshimori, Digital holographic threedimensional imaging spectrometry. Appl. Opt. 52, A388A396, 2013.

[11] M. Adams, Th. Kreis, and W. Jflptner. Particle size and position measurement with digital holography. Proc. of European Symposium on Lasers and Optics in Manufacturing, SPIE, 1997.

[12] Atsushi, S., Yusuke, T., Tomoyoshi, S., Nobuyuki, M., Tomoyoshi, I. Handheld and low-cost digital holographic microscopy., 2012.

[13] P. Ferraro, D. Alferi, S. De Nicola, L. De Petrocellis, A. Finizio and G. Pierattini, Quantitative phase-contrast microscopy by a lateral shear approach to digital holographic image reconstruction, Opt. Lett. 31, 14051407, 2006.

[14] J. A. Herrera Ramrez and J. Garcia-Sucerquia, Digital offaxis holography without zero-order diffraction via phase manipulation. Opt. Commun 277, 259263, 2007. 
Z. Garaguly et al. • The Application of Holographic Image Reconstruction in the Examination of Liquids 